\title{
On the Frequency of Gas Giant Planets in the Metal-Poor Regime
}

\author{
A. Sozzetti ${ }^{1}$, D. W. Latham ${ }^{2}$, G. Torres ${ }^{2}$, B. W. Carney ${ }^{3}$, \\ J. B. Laird ${ }^{4}$, R. P. Stefanik ${ }^{1}$, A. P. Boss ${ }^{5}$, and S. Korzennik ${ }^{2}$ \\ ${ }^{1}$ INAF- Astronomical Observatory of Torino, I-10025 Pino Torinese (TO), Italy \\ email: sozzetti@oato.inaf.it \\ ${ }^{2}$ Harvard-Smithsonian Center for Astrophysics, Cambridge, MA 02138, USA \\ ${ }^{3}$ University of North Carolina at Chapel Hill, Chapel Hill, NC 27599, USA \\ ${ }^{4}$ Bowling Green State University, Bowling Green, OH 43403, USA \\ ${ }^{5}$ Carnegie Institution of Washington, Washington DC 20015, USA
}

\begin{abstract}
We present an analysis of three years of precision radial velocity measurements of 160 metal-poor stars observed with Keck/HIRES. We report on variability and long-term velocity trends for each star in our sample. We identify several long-term, low-amplitude radialvelocity variables worthy of follow-up with direct imaging techniques. We place lower limits on the detectable companion mass as a function of orbital period. None of the stars in our sample exhibits radial-velocity variations compatible with the presence of Jovian planets with periods shorter than the survey duration $(3 \mathrm{yr})$. The resulting average frequency of gas giants orbiting metal-poor dwarfs with $-2.0 \leqslant[\mathrm{Fe} / \mathrm{H}] \leqslant-0.6$ is $f_{p}<0.67 \%$. By combining our dataset with the Fischer \& Valenti (2005) uniform sample, we confirm that the likelihood of a star to harbor a planet more massive than Jupiter within 2 AU is a steeply rising function of the host's metallicity. However, the data for stars with $-1.0 \leqslant[\mathrm{Fe} / \mathrm{H}] \leqslant 0.0$ are compatible, in a statistical sense, with a constant occurrence rate $f_{p} \simeq 1 \%$. Our results usefully inform theoretical studies of the process of giant planet formation across two orders of magnitude in metallicity.
\end{abstract}

Keywords. planetary systems: formation — stars: abundances — stars: statistics — techniques: radial velocities

\section{Introduction}

Fourteen years after the discovery of 51 Pegb (Mayor \& Queloz 1995), the aims of Doppler surveys for planets are now evolving fast. On the one hand, existing surveys are extending their time baseline and/or are achieving higher velocity precision $(\leqslant 1 \mathrm{~m}$ $\mathrm{s}^{-1}$, see for example Lovis et al. 2006), to continue searching for planets at increasingly larger orbital distances (e.g., Fischer et al. 2007) and with increasingly smaller masses (e.g., Mayor et al. 2008). On the other hand, early evidence for a strong relationship between the physical properties of stars and the likelihood that they harbor planets has prompted both theoretical analyses attempting to reconcile the observed trends within the framework of planet formation models as well as renewed experimental efforts to put such trends on firmer statistical grounds and thus thoroughly test the theoretical explanations put forth to explain their existence.

For example, dedicated radial-velocity (RV) surveys of M dwarfs (e.g., Endl et al. 2006), of Hertzsprung gap sub-giants (Johnson et al. 2006), heavily evolved stellar samples belonging to the red-giant branch and clump regions of the H-R diagram (Sato et al. 2003; Setiawan et al. 2005; Lovis \& Mayor 2007; Niedzielski et al. 2007) and early-type dwarfs (Galland et al. 2005) are investigating the predictions for a positive correlation 
between the mass of the host and the occurrence rate and mass of planets (Ida \& Lin 2005).

Another important relationship uncovered so far between planet characteristics and frequencies and host properties is quantified by the strong positive correlation between planet frequency $f_{p}$ and stellar metallicity $[\mathrm{Fe} / \mathrm{H}]$. On the one hand, the observational evidence (Gonzalez 1997; Santos et al. 2004; Fischer \& Valenti 2005) has found theoretical support within the context of the core accretion model of giant planet formation (Ida \& Lin 2004). Doppler surveys biased toward high-metallicity stellar samples (Fischer et al. 2005; Bouchy et al. 2005) have begun in recent years, prompted by the enhanced chances of finding large numbers of planets. On the other hand, the possible bimodality of the $f_{p^{-}}[\mathrm{Fe} / \mathrm{H}]$, with a flat tail for $[\mathrm{Fe} / \mathrm{H}] \leqslant 0.0$ (Santos et al. 2004) might indicate that more than one formation mechanism is at work (e.g., Boss 2002). In order to better characterize the dependence of giant planet frequency in the metal-poor regime, two surveys have started monitoring stellar samples with $[\mathrm{Fe} / \mathrm{H}] \leqslant-0.5$. A southern sample of $\sim 100$ metal-deficient stars is being monitored with HARPS (Santos et al. 2007), while our group (Sozzetti et al. 2006) has focused on a northern sample $\left(\delta \gtrsim-25^{\circ}\right)$ of $\sim 200$ objects. We provide here a summary of the results obtained during our three-year long observing campaign with Keck/HIRES (see Sozzetti et al. (2009a) for details).

\section{Radial-velocity Results}

As described in Sozzetti et al. (2006), all stars are drawn from the Carney-Latham and Ryan samples of metal-poor, high-velocity field stars (e.g., Ryan \& Norris 1991; Carney et al. 1994). Based on a decade-long radial-velocity monitoring with the CfA Digital Speedometers (Latham 1992) none of our program stars showed signs of velocity variation at the 0.5 to $1.0 \mathrm{~km} \mathrm{~s}^{-1}$ level. We selected targets with $V \leqslant 12 \mathrm{mag}, T_{\text {eff }} \leqslant 6000$ $\mathrm{K}$, and $-2.0 \lesssim[\mathrm{Fe} / \mathrm{H}] \leqslant-0.6$.

All observations were collected with HIRES and its $\mathrm{I}_{2}$ cell (except for one Iodine-free template exposure per target) on the Keck 1 telescope (Vogt et al. 1994). To extract the RV information from each star+iodine spectrum, we perform a full spectral modeling which includes the reconstruction of the asymmetries, spatial and temporal variations in the HIRES instrumental profile at the time of observation. Our algorithm follows a procedure based on the methodology developed for the AFOE spectrograph (Korzennik et al. 2000), and adapted for the processing of HIRES spectra, as described in Sozzetti et al. (2009b). The median RV uncertainty for the stars in our sample is $\sigma_{\mathrm{RV}} \simeq 9 \mathrm{~m} \mathrm{~s}^{-1}$.

All stars were probed for excess variability, based on the close relative agreement of three statistical tests ( $F$-test, $\chi^{2}$-test, and Kuiper's test). Nine stars were identified as variables based on the above criteria. These nine objects (HD 7424, G197-45, G 237-84, G 63-5, G 135-46, HD 192718, HD 210295, G 27-44, and G 28-43) all exhibit an RV scatter in the measurements at least four times larger than the nominal average internal error.

For six of the above mentioned objects, the RV data were better described by a linear slope, indicating the presence of a massive companion orbiting with a period greatly exceeding the duration of the observations ( $\gg 3 \mathrm{yr}$ ). As for the other three variables (HD 210295, G 27-44, and G 28-43), the RV residuals were not significantly improved after fitting for a linear trend. The three stars clearly exhibit significantly non-linear RV variations (see Sozzetti et al. (2009a) for details). Both the long-term, low-amplitude radial-velocity variables as well as those showing non-linear trends are the objective of dedicated follow-up work with direct imaging techniques at infrared wavelengths, which will be presented elsewhere. 

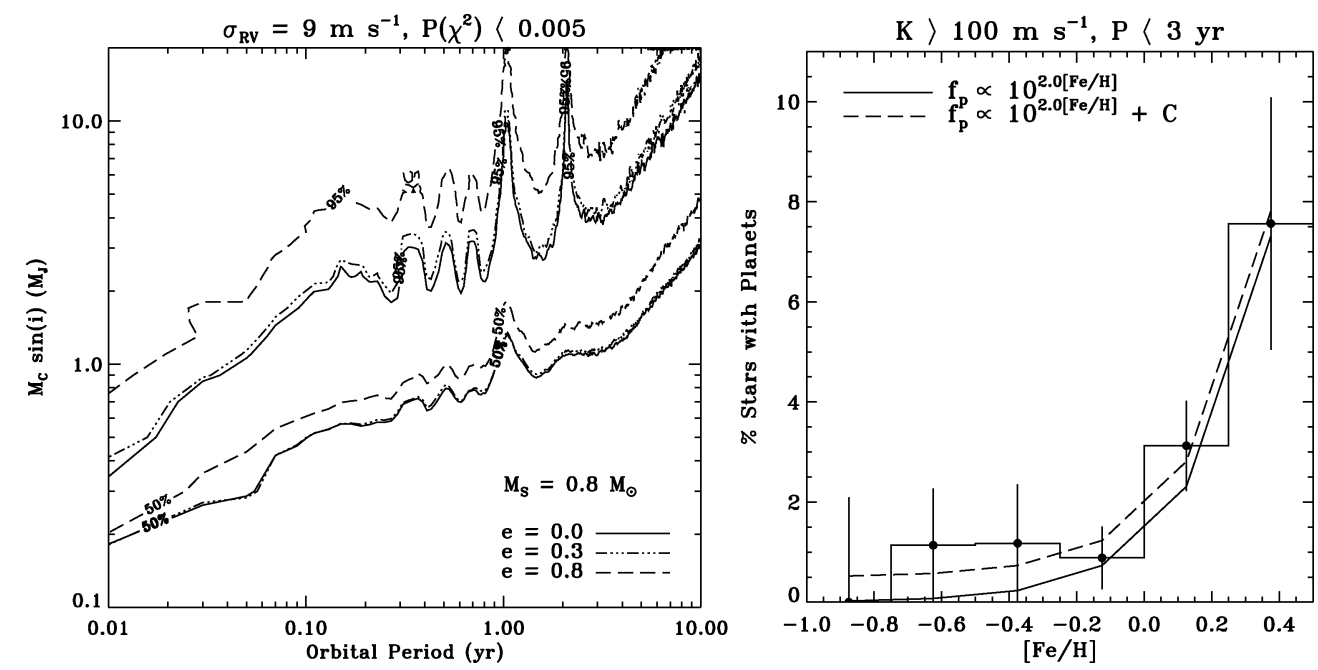

Figure 1. Left: Survey completeness for companions of given mass, orbital period, and eccentricity. The limits shown are for $50 \%$ and $95 \%$ completeness and for three realizations with different values of $e$. Right: Percentage of planet hosting stars as a function of metallicity $(0.25$ dex bins) for the sample constructed combining our survey stars with those of Fischer \& Valenti (2005). The increasing trend in the fraction of stars with planets as a function of metallicity is well fitted with a power law, but the data are compatible with a constant occurrence rate $f_{p} \simeq 1 \%$ for $[\mathrm{Fe} / \mathrm{H}] \leqslant 0.0$.

\section{Survey Completeness}

The quantitative determination of the sensitivity of an RV survey such as ours to planetary companions of given mass and period relies upon detailed numerical simulations of synthetic datasets of RV observations of a population of planetary systems of varying orbital properties and masses. The ability to recover, with a given level of statistical significance, the presence of a planetary signal or not translates into lower limits on the detectable (minimum) companion mass as a function of e.g. period and eccentricity (for an assumed mass of the central star). For this purpose, we utilized a statistical approach based on $\chi^{2}$ - and $F$-tests to detect excess residuals above an assumed level of Gaussian noise. As a result of this exercise, our survey would have detected, with a $99.5 \%$ confidence level, over $95 \%$ of all companions on low-eccentricity orbits with velocity semi-amplitude $K \gtrsim 100 \mathrm{~m} \mathrm{~s}^{-1}$, or $M_{p} \sin i \gtrsim 4.1 M_{\mathrm{J}}(P / y r)^{(1 / 3)}$, for orbital periods $P \leqslant 3 \mathrm{yr}$ (left panel of Figure 1).

None of the stars in our sample exhibits radial-velocity variations compatible with the presence of Jovian planets with periods shorter than the survey duration. The resulting average frequency of gas giants orbiting metal-poor dwarfs with $-2.0 \leqslant[\mathrm{Fe} / \mathrm{H}] \leqslant-0.6$ is $f_{p}<0.67 \%$ (see Sozzetti et al. (2009a) for details).

\section{Discussion}

We do not detect any massive planets $\left(M_{p} \sin i \gtrsim 1-4 M_{J}\right)$ within 2 AU of metalpoor stars with $-2.0 \leqslant[\mathrm{Fe} / \mathrm{H}] \leqslant-0.6$, and constrain their occurrence rate to be no larger than $f_{p} \simeq 1 \%$. All long-period trends identified in our survey are compatible with being induced by brown dwarf or stellar companions. We examine the implications of this null result in the context of the observed correlation between the rate of occurrence of giant planets and the metallicity of their main-sequence solar-type stellar hosts. 
By combining our dataset with the Fischer \& Valenti (2005) uniform sample, we confirm that the likelihood of a star to harbor a planet more massive than Jupiter within 2 AU can be expressed as a quadratic function of the host's metallicity (right panel of Figure 1). These findings appear to be circumstantial evidence in favor of the core accretion mechanism of giant planet formation (Pollack et al. 1996). However, the data for stars with $-1.0 \leqslant[\mathrm{Fe} / \mathrm{H}] \leqslant 0.0$ are compatible, in a statistical sense, with a constant occurrence rate $f_{p} \simeq 1 \%$. This evidence could be read as supportive of the alternative disk instability model (Boss, 2000). However, though very useful, our results are not resolutive, and a number of observational avenues should be pursued (e.g., lowering the mass sensitivity threshold, increasing the sample sizes, and extending the time baseline of planet surveys) to expand and improve the statistics and thus further constrain proposed models.

\section{Acknowledgements}

A.S. gratefully acknowledges for partial support the Kepler mission (NASA Cooperative Agreement NCC 2-1390) and the Italian Space Agency (Contract ASI-Gaia $\mathrm{I} / 037 / 08 / 0)$.

\section{References}

Boss, A. P. 2000 ApJ, 536, L101

Boss, A. P. 2002, ApJ, 567, L149

Bouchy, F., et al. 2005, A\&\&A, 444, L15

Carney, B. W., et al. 1994, AJ, 107, 2240

Endl, M., et al. 2006, ApJ, 649, 436

Fischer, D. A. \& Valenti, J. 2005, ApJ, 622, 1102

Fischer, D. A., et al. 2005, ApJ, 620, 481

Fischer, D. A., et al. 2007, ApJ, 675, 790

Galland, F., et al. 2005, A\&A, 444, L21

Gonzalez, G. 1997, MNRAS, 285, 403

Korzennik, S. G., et al. 2000, ApJ, 533, L147

Ida, S. \& Lin, D. N. C. 2004, ApJ, 616, 567

Ida, S. \& Lin, D. N. C. 2005, ApJ, 626, 1045

Johnson, J. A., et al. 2006, ApJ, 652, 1724

Latham, D. W. 1992, ASP-CS, 32, 110

Lovis, C., et al. 2006, Nature, 441, 305

Lovis, C. \& Mayor, M. 2007, A\&A, 472, 657

Mayor, M. \& Queloz, D. 1995, Nature, 378, 355

Mayor, M., et al. 2008, A\& A, 493, 639

Niedzielski, A., et al. 2007, ApJ, 669, 1354

Pollack, J. B., et al. 1996, Icarus, 124, 62

Ryan, S. G. \& Norris, J. E. 1991, AJ, 101, 1835

Santos, N. C., Israelian, G., \& Mayor, M. 2004, A\&A, 415, 1153

Santos, N. C., et al. 2007, A\&A, 474, 647

Sato, B., et al. 2003, ApJ, 597, L157

Setiawan, J., et al. 2005, A\&BA, 437, L31

Sozzetti, A., et al. 2006, ApJ, 649, 428

Sozzetti, A., et al. 2009a, ApJ, 697, 544

Sozzetti, A., et al. 2009b, ApJ, 691, 1145

Vogt, S. S., et al. 1994, Proc. SPIE, 2198, 362 